\title{
Gedenkrede für Luise Abramowski im Akademischen Festakt, Tübingen, 23. Januar 2019
}

„Dogmengeschichte und Literarkritik“ - mit diesen Worten hat Luise Abramowski einen autobiographischen Beitrag überschrieben, der kurz nach der letzten Jahrtausendwende in einem Sammelwerk eher selbstreferentieller Texte damals zeitgenössischer Kirchenhistoriker veröffentlicht wurde ${ }^{1}$. Die Überschrift „Dogmengeschichte und Literarkritik“ ist treffend und ihre Autorin hat, wie ich bezeugen kann, immer wieder einmal erwogen, sie als Titel über Veröffentlichungen zu setzen, beispielsweise über ihre ins Englische übersetzten Aufsätze². Die Überschrift „Dogmengeschichte und Literarkritik“ ist treffend, weil sie tatsächlich das Spezifikum der Tübinger Ordinaria beschreibt, zu deren akademischem Gedächtnis wir uns versammelt haben.

Auch wenn die Auswahl in dem Sammelband „Kirchengeschichte als Autobiographie“, in dem „Dogmengeschichte und Literarkritik“ veröffentlicht wurde, etwas beliebig wirkt, reicht es, Kollegen-Namen zu nennen, um zu erkennen, dass in der Kombination beider Zugriffe auf Vergangenheit eine Pointe (aber natürlich auch nur eine Pointe) der Arbeiten meiner akademischen Lehrerin im Vergleich zu ihren Altersgenossen und deren Lehrern im Fache lag. Wilhelm Schneemelcher und Friedhelm Winkelmann veröffentlichen im selben Band und repräsentieren die Berliner Traditionen Hans Lietzmanns, also die Traditionen einer Kombination von Textedition, dogmengeschichtlicher Detailarbeit und mehr oder weniger umfangreichen erzählerischen Entwürfen ${ }^{3}$. Robert Stupperich erwähnt dankbar Karl Holl, einen anderen großen Berliner Kirchenhistoriker, bis auf den heutigen Tag Leitstern mancher Reformationshistoriker ${ }^{4}$. Luise Abramowski gehörte in keine dieser Traditionslinien. Im genannten Beitrag verschweigt sie, wer ihre überraschende Kombination einer

1 L. Abramowski, Dogmengeschichte und Literarkritik, in: D. Meyer (Hg.): Kirchengeschichte als Autobiographie. Ein Blick in die Werkstatt zeitgenössischer Kirchenhistoriker, Bd. 2 (Schriften des Vereins für Rheinische Kirchengeschichte 154), Köln 2002, 1-15. - Die Tübinger Worte für Luise Abramowski wurden lediglich durch Fußnoten ergänzt, der Charakter der mündlichen Rede blieb bewahrt. 2 L. Abramowski, Formula and Context. Studies in Early Christian Thought, Variorum Collected Studies Series, Hampshire/Brookfield 1992.

3 W. Schneemelcher, Rückblicke, Erinnerungen und Betrachtungen, in: D. Meyer (Hg.): Kirchengeschichte als Autobiographie. Ein Blick in die Werkstatt zeitgenössischer Kirchenhistoriker, Bd. 2 (Schriften des Vereins für Rheinische Kirchengeschichte 154), Köln 2002, 257-326 sowie Friedhelm Winkelmann, Als Kirchenhistoriker in der Berliner Akademie der Wissenschaften, in: ebd., 367-406. 4 R. Stupperich, Mein Lebensweg von Moskau und vom Ural bis Westeuropa, in: D. Meyer (Hg.): Kirchengeschichte als Autobiographie. Ein Blick in die Werkstatt zeitgenössischer Kirchenhistoriker, Bd. 1 (Schriften des Vereins für Rheinische Kirchengeschichte 138), Köln 1999, 371-396. 
Zugriffsweise der historischen und systematischen Theologie mit einer Methode der exegetischen, vor allem der deutschen alttestamentlichen Wissenschaft als Lehrer grundgelegt hat. Ihre Autobiographie beginnt, ganz wie es ihre nüchterne, ostpreußisch geprägte Art war, mit dem Satz: „Geboren 8. Juli 1928 in Ostpreußen, Schuljahre in Riga, Lyck (Ostpreußen) und Potsdam. Dort Abitur 1946“5. Man hätte es durchaus ausführlicher formulieren können und die ersten achtzehn Lebensjahre durchaus detaillierter beschreiben können, mindestens in einem autobiographischen Essay, und das zweimalige „Ostpreußen“ fällt in der dürren Aufzählung durchaus auf. Und auch Riga war ihr eigentlich wichtig und sie erzählte gelegentlich von der Armut im reformierten Pfarrhaus und vom Neid auf den Reichtum der lutherischen Amtskollegen ihres Vaters. Anlässlich ihres achtzigsten Geburtstags exegesierte sie für die festliche Versammlung im Speisesaal des Tübinger Stifts anstelle des wissenschaftlichen Vortrags eine im Antiquariat erworbene Postkarte in die lettische Hauptstadt nach allen Regeln der Kunst ${ }^{6}$.

Über all das steht im erwähnten Beitrag unter der Überschrift „Dogmengeschichte und Literarkritik“ praktisch nichts zu lesen, „weil ich es zu langweilig fand für mich“. So hat Frau Abramowski mir handschriftlich in ihrer klaren, feinen Handschrift auf den Sonderdruck geschrieben. Was für sie langweilig war, hielt sie selbst nicht schriftlich fest, obwohl es für andere durchaus höchst interessant gewesen wäre. Wenn man verstehen will, warum von Beginn des Studiums in Bonn an die Literarkritik zur Dogmengeschichte trat, muss man die erste Seite der „Geschichte Israels“ aufschlagen, die Martin Noth, seit 1945 Ordinarius für Altes Testament in Bonn, 1950 im Verlag Vandenhoeck \& Ruprecht publizierte. „Für freundliche Hilfe beim Korrekturlesen und für die Anfertigung der Register danke ich Fräulein stud. theol. Luise Abramowski in Bonn, der Tochter meines Freundes Rudolf Abramowski, der 1945 aus seiner Gemeinde und aus seiner wissenschaftlichen Arbeit plötzlich und für uns allzu früh abberufen wurde“7 . Abberufen? „Auf dem Wege zur Deportation in die sowjetische Kriegsgefangenschaft jämmerlich erfroren“, so hat Luise Abramowski mündlich den schlimmen Verlust des sie prägenden Vaters knapp beschrieben, dessen (ihm ursprünglich von Erich Seeberg gestelltes) Dissertationsthema sie mit ihrer 1955 abgeschlossenen Bonner Dissertation aufgenommen und bearbeitet hat ${ }^{8}$. Martin Noth hat mit Hilfe der

5 Abramowski, Dogmengeschichte und Literarkritik, 1.

6 R. Abramowski/E. Cube, Chronik der deutsch-reformierten Gemeinde in Riga, hg. im Auftrag des Presbyteriums, Göttingen 1933.

7 M. Noth, Geschichte Israels, Göttingen 1950, 5.

8 L. Abramowski, [Vorwort zu:] H. C. Brennecke, Bibliographie Rudolf Abramowski, in: H. C. Brennecke/E. L. Grasmück/C. Markschies (Hgg.): Logos. Festschrift für Luise Abramowski zum 8. Juli 1993 (Beihefte zur Zeitschrift für die neutestamentliche Wissenschaft 67), Berlin 1993, 633 (Bibliographie: 634-640). Zur Geschichte ihres Dissertationsthemas: L. Abramowski, Untersuchungen zum Liber Heraclidis des Nestorius (Corpus Scriptorum Christianorum Orientalium 242 = Subsidia 22), Louvain 1963, $11 \mathrm{f}$. 
literarkritischen Methode bekanntlich seine Überlieferungsgeschichte des Pentateuch entwickelt und seine Hypothese eines deuteronomistischen Geschichtswerks formuliert. Man sieht an einem Studienfreund von Luise Abramowski, an Rudolf Smend, wie sehr solche Bonner alttestamentlichen Anregungen auch anderswo bis in die Gegenwart gewirkt haben ${ }^{9}$. Ich will aber nun nicht ausführlicher über diese Dissertation sprechen, die ein erster Beleg für die Bedeutung der Literarkritik für die Dogmen- und Theologiegeschichte ist: Der sogenannte Liber Heraclidis kann dann und nur dann als Quelle für die Rekonstruktion der Theologie des Nestorius herangezogen werden, wenn man den komplexen Wachstumsprozess des uns heute in einer Handvoll syrischer Handschriften überlieferten Textes in Anschlag bringt; Alois Kardinal Grillmeier hat vor vielen Jahren die gründlichen Untersuchungen von Frau Abramowski zu den drei Etappen des Wachstums auf zweieinhalb Seiten knapp zusammengefasst ${ }^{10}$. Mir begegnete diese Form der Kombination von Dogmengeschichte und Literarkritik als Tübinger Student in den späten achtziger Jahren nicht in der syrischen Lektüre, die Frau Abramowski bis fast zur Jahrtausendwende regelmäßig hielt (dort lasen wir die Didascalia apostolorum in einer gerade erschienenen Edition ${ }^{11}$ und nicht den Liber Heraclidis), sondern in der Vorlesung zur Dogmengeschichte, die an vier Nachmittagen jeweils fünfundvierzigminütig gehalten wurde. Aus den Lehrveranstaltungen erwuchsen ihre Aufsätze und in die Lehrveranstaltungen wurden diese Aufsätze wieder im Modus des Selbstreferates hineingetragen. Sowohl in den Vorlesungen „Dogmengeschichte I/1“ als auch „Dogmengeschichte I/2 (Antike nach Chalzedon)“ und ebenso im Spezial-Kolleg „Gnosis“ erlebte man ihre spezifische Kombination von „Dogmengeschichte und Literarkritik“. Ein einziges Beispiel: Frau Abramowski hatte in dem häresiologischen Kompendium des stadtrömischen Theologen Hippolyt (mir sei einmal diese vereinfachende Charakterisierung gestattet) einen ,gnostischen Logostheologen“ als Redaktor eines Quellenkomplexes entdeckt ${ }^{12}$, also wieder drei Ebenen - Hippolyt, gnostisches Sondergut und Logosredaktor - eines literarischen Wachstums hervorgehoben und mit ihrem kleinen grünen Stummelbleistift in der deutschen Übersetzung des Hippolyt die entsprechenden Abschnitte mit den Buch-

9 R. Smend, Martin Noth (1902-1968), in: ders., Kritiker und Exegeten. Portraitskizzen zu vier Jahrhunderten alttestamentlicher Wissenschaft, Göttingen 2017, 825-846.

10 A. Kardinal Grillmeier, Jesus der Christus im Glauben der Kirche. Bd. 1 Von der Apostolischen Zeit bis zum Konzil von Chalcedon (451), 2., verbesserte Aufl., Freiburg u. a. 1982, 708-710.

11 The Didascalia Apostolorum in Syriac, Vol. I Chapters I-X, ed. by A. Vööbus (Corpus Scriptorum Christianorum Orientalium 401 = Scriptores Syri 175), Louvain 1979 bzw. Vol. II Chapters XI-XXVI, ed. by A. Vööbus (Corpus Scriptorum Christianorum Orientalium 407 = Scriptores Syri 179), Louvain 1979. - Das von Frau Abramowski reich annotierte Handexemplar, das in dieser Übung des Sommersemesters 1986 verwendet wurde, befindet sich inzwischen in meinem Besitz.

12 L. Abramowski, Ein gnostischer Logostheologe. Umfang und Redaktor des gnostischen Sonderguts in Hippolyts „Widerlegung der Häresien“, in: dies., Drei christologische Untersuchungen (Beihefte zur Zeitschrift für die neutestamentliche Wissenschaft 45), Berlin/New York 1981, 18-62. 
staben „R“ für Redaktor und „T“ für Tradition markiert; der nicht markierte Rest war Hippolyt, ihrer Ansicht nach jedenfalls ${ }^{13}$.

Bevor ich meine akademische Lehrerin als Kirchenhistorikerin im Geiste von Martin Noth zurechtstilisiere, sollte ich wenigstens noch anfügen, wie sehr ihr Bonner akademischer Lehrer Ernst Bizer und dessen sehr spezifische Sicht auf die Anfänge der Reformation wie der Pointen der Theologie Martin Luthers ihre Arbeit prägte immer wieder gern hat sie Lutherpredigten gelesen und damit das Problem der Identifikation von „Hauptschriften“ des Reformators durch die Schule Karl Holls mehr indirekt zum Ausdruck gebracht, immer wieder hat sie auch in das Neue Testament und in die Theologiegeschichte des Mittelalters ausgegriffen, nicht immer zum Vergnügen ihrer Kollegen. ${ }^{14}$ So viele Jahre nach ihrem Tode darf man vielleicht auch anfügen, dass sie zeitlebens trotz allen Interesses an Martin Luther eine nüchterne reformierte Christin war, die antiochenische Differenzierung zwischen göttlichen und menschlichen Seiten Jesu Christi auch in ihrer Gegenwart theologisch für die überzeugendere Form der christologischen Reflexion hielt und die alexandrinische Tradition ihr eher ferner lag $^{15}$. Sie war, wie es Ostpreußen eben waren ${ }^{16}$, den Menschen herzlich zugetan ${ }^{17}$, konnte aber auch schroff werden und war verletzbar vor allem durch die Herabsetzung, die man im letzten Jahrhundert Frauen im akademischen Betrieb entgegenbrachte. Den Tübinger Ruf (inzwischen darf man es vielleicht öffentlich machen), hat sie im Jahre 1974 wider eigenes Erwarten nur erhalten, weil ein prominenter, von ihrer Gelehrsamkeit beeindruckter Systematiker der Fakultät beim zuständigen Referenten des Ministeriums in Stuttgart intervenierte und der spätere Generalsekretär des Stifterverbandes und Berliner Wissenschaftssenator die Liste der

13 Es handelte sich um: Des heiligen Hippolytus von Rom Widerlegung aller Häresien. Aus dem Griechischen übersetzt von K. Graf Preysing (Bibliothek der Kirchenväter, 1. R. 40) München 1922; inzwischen im Internet zugänglich unter: https://bkv.unifr.ch/works/116/versions/134/divisions/103673 (letzter Zugriff am 15.11.2020).

14 Entsprechende Nachweise finden sich in den beiden Bibliographien: C. Markschies, Bibliographie Luise Abramowski, in: H. C. Brennecke/E. L. Grasmück/C. Markschies (Hgg.): Logos. Festschrift für Luise Abramowski zum 8. Juli 1993 (Beihefte zur Zeitschrift für die neutestamentliche Wissenschaft 67), Berlin 1993, 619-632 sowie Bibliographie Luise Abramowski, Zeitschrift für antikes Christentum 12 (2008) 5-9.

15 Das merkte man vor allem dann, wie ich in meiner Predigt anlässlich ihrer Beerdigung am 14.11.2014 auf dem Tübinger Bergfriedhof gesagt habe, „wenn sie sich ein Herz fasste und immer noch ganz aufgeregt die Kanzel der Tübinger Stiftskirche erkletterte, um zu predigen“ im Akademischen Gottesdienst.

16 Da sie mehrfach recht brüsk abwies, „Doktormutter“ zu sein, und stets „Professor“ genannt werden mochte, nicht „Professorin“ (sie wollte, wie sie mir einmal sagte, den Beruf ihrer männlichen Kollegen ergreifen und nicht einen eigenständigen in einer weiblichen Form), wäre es ihr nicht recht gewesen, hier die weibliche Form „Ostpreußin“ anzufügen.

17 Insbesondere Studentinnen, die während des Studiums ein Kind bekamen, unterstützte sie ebenso liebenswürdig wie großherzig. 
hohen Tübinger Fakultät kippte und den primo loco gesetzten Kollegen nicht berief ${ }^{18}$. Tempi passati. Auch die Emeritierung von Frau Abramowski am Ende des Wintersemesters 1994/1995 liegt schon wieder fast ein Vierteljahrhundert zurück.

Nicht alles, was Luise Abramowski vollenden wollte, ist angesichts ihrer instabilen Gesundheit in den letzten Lebensjahren schon vollendet - so warten Beiträge für das von Theresia Hainthaler herausgegebene Werk „Jesus der Christus im Glauben der Kirche" noch auf ihre Publikation mit den entsprechenden Bänden. Zu den vorbereitenden Materialien, die Frau Abramowski, gründlich wie stets und dem überhasteten Vielschreiben gänzlich abhold, in Angriff nahm, gehört ein Manuskript der syrischen Fragmente einer zentralen Schrift antiochenischer Theologie, des als Ganzem verlorenen Buches De incarnatione des Theodor von Mopsuestia. Auf dieses Manuskript, seine Geschichte und die Kontexte möchte ich kurz noch eingehen, weil die Zusammenhänge für die Autorin des Manuskripts höchst charakteristisch sind. Seit längerem war bekannt, dass Luise Abramowski an der syrischen Überlieferung von De incarnatione arbeitete. Etwas verwundert musste die Fachwelt dann aber zur Kenntnis nehmen, dass vor inzwischen fast zwanzig Jahren ein junger Göttinger Doktoratsstudent meinte, unter dem Buchtitel „Theodor von Mopsuestia, De Incarnatione“19 ausschließlich die griechischen und lateinischen Fragmente übersetzen und originalsprachlich erneut edieren zu können. Syriaca sunt, non leguntur. Keine syrisch geschriebene Zeile befindet sich in diesem Buch, das doch ein bis in die Katastrophen des zwanzigsten Jahrhunderts noch vollständig in syrischer Übersetzung und beträchtlichen syrischen Fragmenten erhaltenes antikes Werk behandelt. ${ }^{20} \mathrm{Zu}$ Lebzeiten erschienen die Vorarbeiten von Luise Abramowski für die Edition der syrischen

18 So mir gegenüber der spätere Generalsekretär des Stifterverbandes für die Deutsche Wissenschaft, Manfred Erhardt, damals Referent für die Universität Tübingen im Kultusministerium Baden-Württemberg, mündlich bei einem Gespräch im Februar 2006 in Berlin. Erhardt hat diese Sicht noch einmal bei einem Gespräch im Oktober 2019 bekräftigt.

19 T. Jansen, Theodor von Mopsuestia, De incarnatione. Überlieferung und Christologie der griechischen und lateinischen Fragmente einschließlich Textausgabe (Patristische Texte und Studien 65), Berlin 2009. - Im Vorwort wird Luise Abramowski gedankt „für ein sehr motivierendes Gespräch, weiterführende Korrekturen und Hinweise sowie ihre Unterstützung bei der Veröffentlichung dieser Studie“ (aaO., VII).

20 Der Kollege Ekkehard Mühlenberg als Betreuer der Dissertation wies mich bei einem Gespräch nach dem Tübinger Festakt 2019 darauf hin, dass in der Klärung stemmatischer Verhältnisse durch die Arbeit ein Wert an sich liege und Frau Abramowski durchaus der Meinung gewesen sei, man könne nur die griechische und lateinische Seite der Überlieferung der Schrift Theodors studieren. Der hier vorgetragene kritische Einwand wird freilich nicht allein von mir geltend gemacht: „Leider scheint der Autor [sc. Jansen] des Syrischen (und des Armenischen) nicht mächtig, so dass er die Forschungsergebnisse der Grande Dame der deutschen Syrologie, Luise Abramowski, ungeprüft übernehmen muss. Überhaupt ist der Ausfall der syrischen Tradition, und zwar sowohl der monophysitischen wie auch der diophysitischen, im Falle Theodor aufs heftigste zu beklagen“ (P. Bruns, Rez. Till Jansen: Theodor von Mopsuestia, De incarnatione, Sehepunkte 11 (2011), Nr. 3 (http://www.sehepunkte.de/2011/03/17540.html; letzter Zugriff am 15.11.2020). Ganz ähnlich äußert sich in seiner Rezension der Dissertation S. Gerber, 
Traditionen und ihre Übersetzung nicht mehr. Erst jetzt kann an die Seite der durchaus verdienstvollen Neuedition der griechischen und lateinischen Überlieferung durch Jansen und der Fragmente bei Leontius von Byzanz durch Daley ${ }^{21}$ eine neue Edition der beiden Reihen syrischer Fragmente der Schrift De incarnatione treten. Frau Abramowski kam auf dieses Thema ursprünglich im Zuge ihrer Vorarbeiten für die Ausgabe der berühmten syrisch-miaphysitischen Handschrift Brit. Mus. Add. 12156 für das Löwener „Corpus Scriptorum Christianorum Orientalium“, zusätzlich herausgefordert durch die Aufgabe, ein Manuskript des römischen Jesuiten Raimund Köbert vom römischen Päpstlichen Bibelinstitut ${ }^{22}$ mit Übersetzungen der Fragmente für Kardinal Grillmeiers erwähntes Werk kritisch durchzusehen. Ihre handschriftlichen Vorarbeiten für eine grundlegende Revision der deutschen Übersetzung von Köbert übertrug der damalige Assistent 1990 erstmals in eine digitale Form (ein Lehrstuhlsekretariat war Frau Abramowski bei den Tübinger Verhandlungen nicht zugebilligt worden) ${ }^{23}$. Dank der Hilfe der Gertrud-und-Alexander-Böhlig-Stiftung, dank der Arbeit von Alexander Markus Schilling, Volker Drecoll und Hanns Christof Brennecke ist, was ich als Assistent einst in den Computer schrieb, nun zu einer veritablen Edition von syrischem Text und deutscher Übersetzung samt griechischen und lateinischen Parallelen im Apparat auf über fünfzig Seiten angewachsen. Angesichts der langen Veröffentlichungsdauer kann man nur darauf hinweisen, dass gut Ding bekanntlich Weile haben will, und hoffen, dass die hier nun vorgelegte Fassung einigermaßen dem entspricht, was sich Luise Abramowski zu Lebzeiten vorgestellt hatte.

Der Band christologischer Untersuchungen, in dem diese erweiterte Edition der syrischen Fragmente Theodors erscheint, ist ein Zeichen des bleibenden Dankes von Volker Drecoll, Hanns Christof Brennecke und mir an unsere hochverehrte akademische Lehrerin bzw. Lehrstuhlvorgängerin, deren methodische Strenge und rigorose Gelehrsamkeit wir noch immer gelegentlich als Druck empfinden, aber deren attraktive Seiten uns ebenso immer bewusst sind, um ihre Worte über den eigenen Vater

in: Zeitschrift für Antikes Christentum 15 (2011) 539-542. Weitere Belege für diese Einschätzung könnten angefügt werden.

21 B. E. Daley, Leontius of Byzantium. Complete Works. Edited and Translated, with an Introduction (Oxford Early Christian Texts), Oxford 2017.

22 W. R. Mayer, In memoriam: P. Raimund Köbert SJ (1903-1987), Orientalia. Nova Series 57 (1988) 212-217.

23 In der „editorischen Notiz des Bearbeiters“ Alexander Markus Schilling (unten, S. 37 f.) ist diese Fassung als „Typoskript $h$ )“ bezeichnet. Die Datumsangabe „(1. Manuskriptstufe, 30. Dezember 1990)“ wurde von mir seinerzeit gegenüber der handschriftlichen Vorlage eingefügt und sollte den damit erfolgten Beginn der elektronischen Erfassung von weiteren digitalen Bearbeitungsstufen unterscheiden helfen. Bis 1994 nahm Frau Abramowski aber nur noch handschriftliche Ergänzungen in diesem Ausdruck vor, die sie nicht mehr in die elektronische Fassung der Datei einfügen ließ. Der handschriftlich korrigierte Ausdruck (das Typoskript) gibt also vermutlich ihren Überlegungsstand aus dieser Zeit wieder. 
geringfügig zu modifizieren ${ }^{24}$. „Was wissen Sie über Vööbus als Editor?“, lautete die erste Frage in der erwähnten Syrisch-Übung über die Didascalia Apostolorum an eine Handvoll Studierende mittleren Semesters, die natürlich nichts, aber auch gar nichts über den estnischen evangelischen Theologen, Kirchenhistoriker und Orientalisten Arthur Vööbus und seine Verdienste als Editor wussten. Erst am Ende des Semesters, da wussten die Teilnehmenden nicht nur viel über eine bemerkenswerte spätantike Kirchenordnung, sondern auch etwas über Vööbus als Editor ${ }^{25}$. Für solche aus heutiger Perspektive wenig von didaktischer Kunst geprägten, aber höchst intensiven und einprägsamen Einführungen wie die hier beispielhaft vorgestellte sind vermutlich nicht nur die drei genannten Kirchenhistoriker Luise Abramowski bis heute dafür außerordentlich dankbar. Viele haben Grund, ihr bleibend dankbar zu sein.

Ich illustriere, weil allzu viel Dank der magistra (wie Ernst Ludwig Grasmück zu sagen pflegte) gar nicht recht gewesen wäre, diese Zusammenhänge abschließend mit einer Anekdote. Als der schöne Vorlesungssaal im Tübinger Theologicum kurz vor der Einweihung stand, besuchte Frau Abramowski den nahezu fertiggestellten Raum und inspizierte vor allem das Vorlesungspult. Der nicht wenig stolze Architekt wartete gespannt auf einen freundlichen Kommentar zu dem eindrücklichen oktogonalen Bau und seinen sorgfältig ästhetisch gestalteten Details. Der Kommentar kam auch, aber er fiel ganz anders aus als erwartet: „Wie kann man“, sagte Frau Abramowski damals, „nur ein so unpraktisches Vorlesungspult hier hinstellen. Es lässt sich ja gar nicht in der Höhe verstellen“. So oder ungefähr so sprach sie und das unpraktische Vorlesungspult wurde prompt vom zerknirschten Architekten umgerüstet. Seither kann es in der Höhe verstellt werden - und nach dieser Geschichte werden mir auch die glauben, die das noch gar nicht wussten, dass es mindestens in Tübingen noch viele kaum bekannte gute Gründe gibt, Luise Abramowski dankbar zu sein. Die im Sammelband vorgelegten Aufsätze und Editionen zählen mit Sicherheit zu diesen kaum bekannten oder gänzlich unbekannten Gründen.

24 Abramowski, [Vorwort zu:] H. C. Brennecke, Bibliographie Rudolf Abramowski, 633.

25 Nahezu sicher bin ich mir aber, dass Frau Abramowski uns nichts darüber erzählte, dass Vööbus nicht nur an der estnischen theologischen Fakultät der Universität Tartu arbeitete (ders., The Department of Theology at the University of Tartu. Its Life and Work, Martyrdom and Annihilation [Papers of the Estonian Theological Society in Exile. Scholarly Series 14], Stockholm 1963), sondern auch an der „Luther-Akademie“, einem maßgeblich von Erich Seeberg, dem akademischen Lehrer von Rudolf Abramowski, bestimmten Versuch, wieder eine deutsche theologische Ausbildungsstätte am Ort zu errichten (diese Hochschule wurde 1931 gegründet und schloss 1938; S. Bitter, Umdeutung des Christentums. Der baltische Theologe Erich Seeberg im Nationalsozialismus, in: M. Garleff [Hg.]: Deutschbalten, Weimarer Republik und Drittes Reich, Bd. 1, 2., durchg. u. erg. Aufl., Köln u. a. 2008, [267-296] 275-280). Laut Hinweis von Vööbus im ersten Band seiner Edition der Didaskalie (p. 10*) gehen die Vorarbeiten der Edition auf die Zeit zurück, die er in seiner Heimat verbracht hat. 